\title{
Gradhiva
}

GRADHIV

Revue d'anthropologie et d'histoire des arts

13 | 2011

Pièges à voir, pièges à penser

\section{Récursions chimériques}

De l'anthropomorphisme des robots autonomes à l'ambiguïté de l'image du corps humain

Chimeric Recursion. From the anthropomorphism of autonomous robots to the ambiguity of images of the human body

\section{Joffrey Becker}

\section{(2) OpenEdition}

\section{Journals}

Édition électronique

URL : http://journals.openedition.org/gradhiva/2116

DOI : $10.4000 /$ gradhiva. 2116

ISSN : 1760-849X

Éditeur

Musée du quai Branly Jacques Chirac

Édition imprimée

Date de publication : 18 mai 2011

Pagination : 112-129

ISBN : 978-2-35744-042-5

ISSN : 0764-8928

Référence électronique

Joffrey Becker, «Récursions chimériques », Gradhiva [En ligne], 13 | 2011, mis en ligne le 18 mai 2014, consulté le 01 mai 2019. URL : http://journals.openedition.org/gradhiva/2116 ; DOI : 10.4000/ gradhiva. 2116 



\section{Récursions chimériques}

\section{De l'anthropomorphisme des robots autonomes à l'ambiguiité de l'image du corps humain}

"The combination [of top-down strategy and bottom-up evolution] will create beings that in some ways resemble us, but in other ways are like nothing the world has seen before."

Hans Moravec, Mind children (1988).

Joffrey Becker

Fig. 1 Automate. Câble pivotant. Milieu du $x_{1 X} x^{e}$ siècle. Le fabricant de cet automate est inconnu, il a été utilisé par le forain Rudolf Messerer. Bois peint et sculpté, métal, cheveux. @ BPK, Berlin, Dist. Rmn/image BPK.
L'idée que les humains puissent un jour refuser de partager leur quotidien avec des robots inquiète les roboticiens (Mori 1970; Gelin 2006). Bon nombre d'études menées dans le domaine de l'interaction humain-robot gardent, ainsi, le souci d'évaluer la relation à la machine en matière d'acceptation ou de rejet. Quelques grands rassemblements ont, par ailleurs, été organisés sur la base de cette inquiétude. Ils encouragent chercheurs et industriels à sortir de l'intimité de leurs laboratoires pour aller à la rencontre d'un public non spécialiste, et promouvoir, auprès de lui, une robotique qui serait performante et conciliable avec certaines grandes préoccupations du monde contemporain. Ces préoccupations sont d'ordre divers, et concernent autant l'activité de travail, la défense ou la sécurité, que l'assistance à la personne et la santé. L'assistance de machines autonomes, capables de prendre des décisions et d'agir seules, serait devenue inévitable dans un monde qui prévoit, pour la seconde moitié du xxi siècle, un vieillissement global de sa population. On peut facilement comprendre en quoi ce motif constitue un enjeu d'une importance fondamentale, mais également en quoi le refus des humains de vivre avec ces machines pourrait remettre en question les investissements colossaux, privés et publics, qui sont effectués dans ce domaine. On peut saisir alors tout l'intérêt de ces grands rassemblements; lieux d'échanges et de transferts technologiques, ils forment également de puissants outils de promotion intéressant la presse internationale, et sont l'occasion de mettre en présence des ingénieurs, leurs machines, des curieux de tous âges, et des passionnés de robotique venus du monde entier, autour du souci de construire une identité commune aux humains et aux robots. 


\section{PIÈGES À VOIR, PIÈGES À PENSER}

-

1. Je saisis ici l'occasion pour remercier monsieur Carlo Severi et les membres du projet ANR «Anthropologie de l'art : création, rituel, mémoire » qui m'ont permis d'assister à cet événement. J'adresse également mes remerciements à madame Marika Moisseeff, messieurs Denis Vidal, Michael Houseman, Emmanuel Grimaud; à messieurs Bruno Maisonnier, Jérôme Monceau et au personnel de la société Aldebaran Robotics; à messieurs François Charpillet, Alain Dutech, Nicolas Rougier, et à l'ensemble des chercheurs des équipes MalA et Cortex du LORIA-INRIA, ainsi qu'aux participants du colloque Robots-Hybrides-Cyborgs organisé par messieurs Bernard Andrieu et Didier Manuel.
Dans l'événement que nous allons maintenant aborder, la RoboCup ${ }^{1}$, la mise en relation de machines autonomes avec le public cherche à provoquer, chez le spectateur, une évaluation positive de l'image mobile des machines à travers l'humanité qu'elle tente de figurer. L'image montrée par de tels robots reposerait pourtant moins sur cette continuité espérée, que sur une série d'ambiguités. S'ils sont ainsi pensés comme des architectures complexes capables d'offrir la garantie d'une relation sociale acceptable pour l'utilisateur humain (Brooks 2002; Breazeal 2003), les robots mettent en jeu des procédés de conception se référant à des principes dépassant le corps dont ils tentent d'imiter l'image. À un autre niveau, l'action de ces objets entraîne le spectateur dans une dynamique perceptuelle, où la focalisation de l'attention sur la ressemblance d'un objet avec un corps de référence permet une forme particulière de projection (Gell 1992). Ces robots participeraient dès lors de l'établissement d'un contexte relationnel particulier qui, prenant l'objet mobile pour centre, oriente l'attention sur le déchiffrement de ses actes à partir d'un modèle du même ordre que celui dont il cherche à illustrer les traits. Se concevant ainsi à partir de l'organisation de quelques traits saillants, mais également en tant qu'objets qui orientent la projection d'une interprétation, les robots de la RoboCup nous offrent l'occasion d'une réflexion à partir de la notion de chimère (Severi 2007). On peut certes imaginer que l'écart entre, d'un côté, les modalités de la conception du modèle et, de l'autre, le contenu de l'inférence engageant le spectateur dans une relation avec une machine, fonde une certaine résonance avec le principe formulé par Carlo Severi. Et l'on peut également penser que cet écart participe d'une nouvelle autonomie de l'objet vis-à-vis de l'image qu'il figure. Cette dernière ne serait plus seulement définie à travers les capacités de décision et d'action des objets, mais également à travers la médiation qu'ils peuvent assurer entre le modèle que l'ingénieur tente d'imiter et celui que le spectateur est tenté de projeter. Sous cet angle, les robots n'apparaîtraient plus comme les éléments principaux de la relation mais comme les intermédiaires d'un mouvement récursif allant du corps imité à l'interprétation de cette imitation, telle qu'elle prend le corps pour modèle. À l'image des automates de l'âge classique, les robots constitueraient ainsi des formes de simulation et de dramatisation de la vie, s'offrant dès lors en autant de manifestations matérielles d'une théorie de la personne à travers une renégociation des limites du vivant et de l'intelligence (Riskin 2003).

Après avoir présenté l'événement qui nous servira de fil conducteur, je donnerai quelques détails sur la manière dont sont conçues certaines des machines qui y sont présentées. Cette description m'amènera à considérer le rapport de continuité et d'ambiguïé qui me semble caractériser les relations des humains envers ces objets. J'envisagerai ensuite ce rapport à partir de la description d'un mouvement particulier, accompli par des robots humanoïdes. Nous verrons qu'une autonomie particulière peut émerger de la relation entre la conception du comportement des machines et son observation par les spectateurs. Je conclurai en exposant que ce jeu de projection est lié à l'autonomie même de l'image du corps humain, et qu'il consiste en une forme d'alternance entre l'imitation et l'invention collective.

\section{Éléments de contexte}

Le contexte de cette étude est celui d'une compétition de robotique comme il y en existe un peu partout dans le monde. Créée en 1997, la RoboCup vise à promouvoir des recherches menées dans les domaines de la robotique et de l'intel- 
ligence artificielle à partir d'un problème commun (Kitano 1997). Cette compétition regroupe plusieurs formes de jeux dont un retiendra notre attention. Il s'agit d'un tournoi de football impliquant différentes catégories de robots autonomes, anthropomorphes à divers degrés. Un des buts envisagés par ses organisateurs est la conception, d'ici à 2050, d'une équipe de robots capable de vaincre l'équipe humaine tenante du titre mondial ${ }^{2}$. Mais s'il est bien question de performance, ce tournoi ne présente en réalité que peu de points communs avec le sport. La RoboCup rassemble essentiellement des universitaires du monde entier et, si le niveau de jeu est encore loin d'atteindre celui d'une équipe humaine, les problèmes traités par les jeunes ingénieurs requièrent un haut niveau de compétence. La RoboCup est ainsi une opportunité dont certains participants se saisissent pour valoriser leurs propres travaux et de nombreuses thèses sont d'ailleurs menées dans le cadre de cette compétition.

Le jeu est, plus généralement, un support idéal pour les travaux conduits en robotique et en informatique. Il permet de circonscrire des cadres stricts pour la résolution de problèmes et le développement de technologies performantes qui sont construites a minima, spécialement pour les résoudre. Le cadre de jeu pose néanmoins des problèmes suffisamment compliqués pour intéresser la recherche. Il permet aux chercheurs d'apporter des solutions nouvelles aux difficultés que rencontrent habituellement leurs disciplines, liées notamment à la robustesse du contrôle moteur et au traitement des signaux. L'évaluation d'une technologie à travers un jeu se mesure, par ailleurs, en des termes simples de victoire ou de défaite. Le jeu se définit enfin comme une pratique ancienne en intelligence artificielle et en robotique, qui offre une visibilité certaine aux travaux réalisés en la matière ${ }^{3}$ (Crevier 1997).

Or, si la RoboCup consiste bien en un contexte de ce genre, la façon dont l'action des machines se réfère à celle du corps encourage à une certaine prudence. Si le jeu permet la création d'un cadre spécifique pour le développement technologique, il s'impose surtout comme un objet en soi, dont l'étude permet de mettre en valeur la profondeur des relations qui s'y tissent (Geertz 2005). Gregory Bateson a souligné que l'activité ludique forme un espace paradoxal qui confond la réalité et sa représentation, et consiste en un contexte de métacommunication où les actions "ne désignent pas la même chose que désigneraient les actions dont elles sont des valant pour " (Bateson $1977: 250$ ). Dans le contexte de la RoboCup, ces actions dépendent de deux espaces bien distincts. D'une part, elles varient selon une stratégie développée en privé par chaque équipe, en amont de la partie. Cette stratégie s'appuie sur la manière dont les équipes parviennent à déléguer leur propre intelligence du jeu aux machines, à travers la construction de leurs architectures internes et, dans la plupart des cas, externes ${ }^{4}$. D'autre part, ces actions sont effectuées en public, non par des joueurs humains, mais par des objets. Néanmoins, ces actions excluent les humains des relations habituelles $\mathrm{du}$ jeu pendant le match. Lorsque la partie est ainsi jouée par les machines, les joueurs humains deviennent des spectateurs, voire de bruyants supporters, qui n'ont pas le droit d'intervenir sur les machines en dehors des périodes d'arrêt de jeu. Les arbitres demeurent les seuls humains à pouvoir agir sur le type d'action de jeu des machines au cours du match.

La difficulté à comprendre ces jeux tient, ici, dans l'analogie construite entre les robots et les humains qu'ils figurent dans le contexte de la partie. L'un des traits caractéristiques de la situation d'un joueur ordinaire est qu'il se situe, du fait

\footnotetext{
- $\bullet$

2. La RoboCup cherche par ailleurs à se constituer en un événement suffisamment visible pour créer des liens durables avec l'industrie (Kitano 1997 : 83).

3. L'exemple le plus connu reste les parties opposant la génération d'ordinateurs Deep Thought et le champion du monde d'échecs Gary Kasparov entre 1994 et 1997. Mais le développement d'une informatique à l'appui du jeu, et par extension la compétition des machines et des humains, apparaît dès la fin des années 1940 (Crevier 1997).

4. La plupart des ligues de la compétition imposent aux joueurs de fabriquer non seulement le programme permettant aux objets d'effectuer leur tâche, mais également de confectionner les machines elles-mêmes.
} 
$\mathrm{du}$ cadre de règles dans lequel il agit, dans une tension permanente entre ses dispositions émotionnelles et intentionnelles (Houseman 2003 : 299). Or, nous savons bien que les robots n'ont aucune idée de ce qu'ils font, qu'ils se contentent d'exécuter une tâche selon des modalités précises, dont ils ne peuvent saisir l'enjeu, et qui ne requiert aucune motivation particulière de leur part, sinon celles de leurs opérateurs (Braitenberg 1991 : 2). On peut craindre, ici, qu'une notion de jeu ne se préoccupant guère du comportement des objets ne puisse nous permettre d'envisager le problème que du seul point de vue humain; et donc, de passer à côté de l'essentiel de ce qui se joue à travers la médiation particulière qu'assurent les machines. Il me semble nécessaire, dans un premier temps, de laisser de côté la notion de jeu pour mieux l'intégrer, par la suite, à ce qui constitue le contexte plus général des relations entretenues autour des robots. Si l'on souhaite ainsi mieux saisir la manière dont l'image du corps intervient dans la construction des machines puis l'interprétation de leurs actes, il convient de mettre en valeur les éléments qui caractérisent leurs actes. En effet, si l'anthropomorphisme touche à la forme particulière de certaines machines, il peut être étendu aux différents espaces qu'elles occupent, mais également aux méthodes qui déterminent leurs possibilités d'action.

Les robots présents dans cette compétition adoptent diverses apparences et ne renvoient pas toujours à l'image humaine de manière très claire. Ils consistent en des agents de tailles différentes et de nombre variable, évoluant sur des terrains de tailles distinctes, sur lesquels se disputent des matchs dans différentes ligues, articulant plusieurs compétitions au sein du tournoi. Certains robots n'ont ainsi d'existence que virtuelle. D'autres ne sont pas pourvus de caméra et comptent parfois sur des percepts externalisés qui s'appuient sur l'usage d'un système de contrôle supervisant l'ensemble des actions des agents sur le terrain. Il semble en fait plus simple d'envisager l'anthropomorphisme de ces machines en considérant ce dernier à travers trois aspects. Les robots évoluent d'abord dans un cadre spécifique et leurs actes sont limités aux contraintes liées à cet espace. Ils disposent ensuite d'une forme qui leur permet d'agir efficacement. Cette efficacité dépend, enfin, de la manière dont chaque machine a été programmée. Quelques exemples nous permettront d'en envisager les composantes. Nous

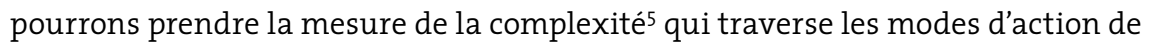
ces curieux robots footballeurs.

\section{Du modèle aux comportements : un jeu avec l'intelligence collective}

(2)

5. J'emploie le terme dans le sens utilisé par C. Severi, c'est-à-dire en tant qu'il connote, à l'appui d'indices visuels particuliers, l'identité d'un énonciateur objectivé (Severi 2009: 16-17).
L'une des ligues de la RoboCup, la Small Size League, constitue l'une des formes de jeu de robots parmi les plus connues. Ce type de jeu est pratiqué dans d'autres compétitions internationales. Il se concentre sur des problèmes liés au contrôle et à la coordination d'agents robotiques placés dans un environnement dynamique. Celui-ci (fig. 2) consiste en une plate-forme d'un peu plus de six mètres sur quatre, recouverte d'un tapis de feutre vert, lui-même traversé des lignes blanches caractéristiques d'un terrain de football. Ce terrain est doté de deux cages et il est surmonté d'un portique sur lequel sont fixées des caméras, celles-ci servant de système de vision. Elles permettent de détecter la position de la petite balle de golf orange utilisée pour la compétition, ainsi que les positions respectives des robots de chaque équipe. Ce système de supervision, placé sous la responsabilité d'un expert humain, renvoie ces informations vers les unités centrales de 


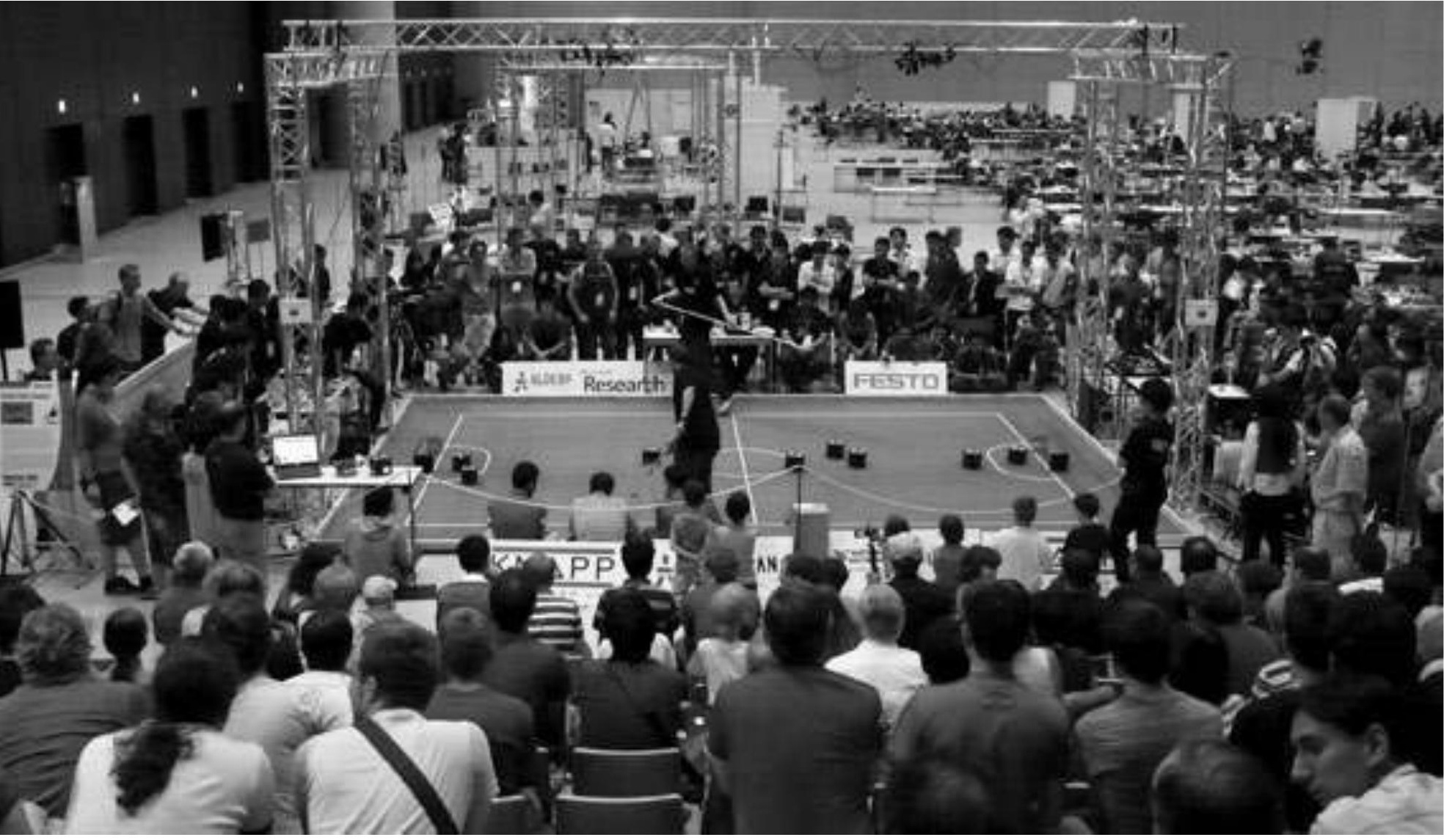

Fig. 2 Vue générale d'un terrain de jeu de la Small Size League, RoboCup 2009, Graz. Photo Joffrey Becker.

chaque équipe, où elles sont analysées et traitées, afin que les commandes soient envoyées de manière automatique vers les machines, par le biais de transmetteurs sans fil.

Chaque match dure dix minutes et chaque équipe dispose de quatre périodes de temps mort. Le reste de la partie suit certaines des règles du football. Ainsi, lorsque la balle franchit les limites du terrain de jeu, on procède à des remises en jeu qui empruntent au vocabulaire ordinaire du football ${ }^{6}$. Les robots peuvent par ailleurs commettre des fautes, et certains de leurs actes sont sanctionnés d'un coup franc direct ou indirect, voire d'un penalty. La règle du jeu veut, ainsi, que le contact entre des agents robotiques, ou l'obstruction d'un agent par un autre, soit considéré comme des comportements à sanctionner par l'arbitre humain. Celuici peut éventuellement attribuer un carton jaune si l'un des agents présents sur le terrain adopte un "comportement antisportif "; s'il est coupable, entre autres choses, d'une violente collision avec un agent adverse, ou encore s'il gêne le travail de l'arbitre en pénétrant dans la zone d'arbitrage, sur la bordure extérieure du terrain. L'agent sanctionné doit alors quitter le terrain pendant deux minutes. Et il doit définitivement abandonner le jeu si l'arbitre a réprimé son comportement d'un carton rouge. En cas d'égalité entre les deux équipes, à la fin du temps réglementaire, on procède à deux périodes de prolongation de cinq minutes, puis à une séance de tirs au but si les équipes n'ont pu se départager.
○

6. On parle par exemple de throwin, de corner kick ou de goal kick. 
-

7. Sans qu'il me soit possible de mettre cette assez vaste collection d'existants en relation avec les systèmes conçus spécifiquement dans le cadre de la RoboCup, j'aimerais noter, ici, qu'elle se fonde à partir de recherches allant des amibes aux humains, en passant notamment par les fourmis ou les rats. Pour un rapide aperçu du bestiaire de la robotique, on se référera à l'ouvrage de $\mathrm{A}$. Guillot et J.-A. Meyer (2008).
Les robots (fig. 5) sont conçus par les roboticiens de chaque équipe et consistent en de petits véhicules cylindriques d'environ dix-huit centimètres de diamètre et quinze centimètres de hauteur, contenant principalement des actionneurs et le matériel électronique qui en assure à la fois l'alimentation, le contrôle, et permet d'acquérir des informations sur l'état de chaque robot. Les machines possèdent ainsi trois ou quatre roues omnidirectionnelles, des moteurs, et un dispositif de contrôle permettant de frapper la balle. Le nombre de robots composant chaque équipe n'est pas limité. Au cours du jeu, il est en revanche fixé à cinq, répartis en quatre joueurs de champ et un gardien de but. Le nombre de changements au cours de la partie est libre. Chaque robot doit porter un numéro qui permette de l'identifier. Les machines arborent également la marque de leur équipe sur le dessus de leur capot afin d'être identifiées par le système central de vision.

Dernier élément du dispositif de jeu, le système de commande permet d'élaborer la stratégie coordonnant l'action des robots sur le terrain de jeu. Celuici s'appuie sur le traitement des signaux vidéo reçus par l'intermédiaire du système de vision central. Il prend alors des décisions en fonction de différents paramètres pertinents, conçus par les programmeurs. Ces décisions sont ensuite traduites en commandes motrices et envoyées vers les robots. Ces systèmes d'intelligence artificielle constituent l'élément clé du dispositif, bien qu'ils se trouvent aux marges du terrain de jeu et qu'ils n'attirent l'attention de personne pendant le match. Et si les équipes communiquent beaucoup sur la manière dont sont conçus matériellement leurs agents, elles en disent en revanche un peu moins sur la manière dont ces derniers s'organisent au cours du jeu. En ce domaine, chaque équipe agit avec les connaissances dont elle dispose. Et l'on peut aisément imaginer que ces connaissances varient considérablement d'une équipe à l'autre. Ainsi, il n'est pas rare de pouvoir observer, au cours de la compétition, des patterns liés au type de situation de jeu rencontré par le système. Le but étant alors de fermer les angles de tir et d'organiser la position de chaque agent en fonction de l'endroit où se trouve la balle, ou son possesseur. Le jeu se résume ensuite à un ensemble de réactions stéréotypées qui consistent en des recherches de fenêtres de tirs, la mise en route de phases de jeu ou des replis défensifs très rapides de l'ensemble des agents. Ici, l'accent est mis sur l'évaluation statistique, par le système, de la meilleure configuration à exploiter afin d'augmenter le score de l'équipe. Ces méthodes habituelles du domaine de l'intelligence artificielle, relatives notamment aux systèmes multi-agents, peuvent être mobilisées par les joueurs humains, lorsqu'ils doivent ordonner la pensée stratégique qu'un tel système est tenu de mettre en œuvre de manière autonome, lors d'un match.

Il n'y a en fait rien d'étonnant à trouver, à la source de certains comportements autonomes des machines, des éléments d'une modélisation des fonctions du vivant. Cette modélisation s'inspire notamment de la psychologie, de l'éthologie ou des neurosciences et n'est pas toujours très soucieuse du genre d'existant dont il s'agit d'imiter la fonction? ${ }^{7}$. Il m'a été donné de rencontrer un roboticien, spécialiste du domaine des systèmes multi-agents qui, au début de sa carrière, a eu l'occasion de participer à ce type de compétitions. Il s'en est aujourd'hui détourné, préférant à ces systèmes très centralisés, des recherches sur des agents réactifs ne recouvrant qu'une perception très locale de leur environnement. Ce chercheur a néanmoins travaillé à lélaboration de systèmes de commande qui, bien que maintenant assez anciens, sont proches de ceux que l'on rencontre aujourd'hui dans ces compétitions. Voici ce qu'il m'en a dit : 


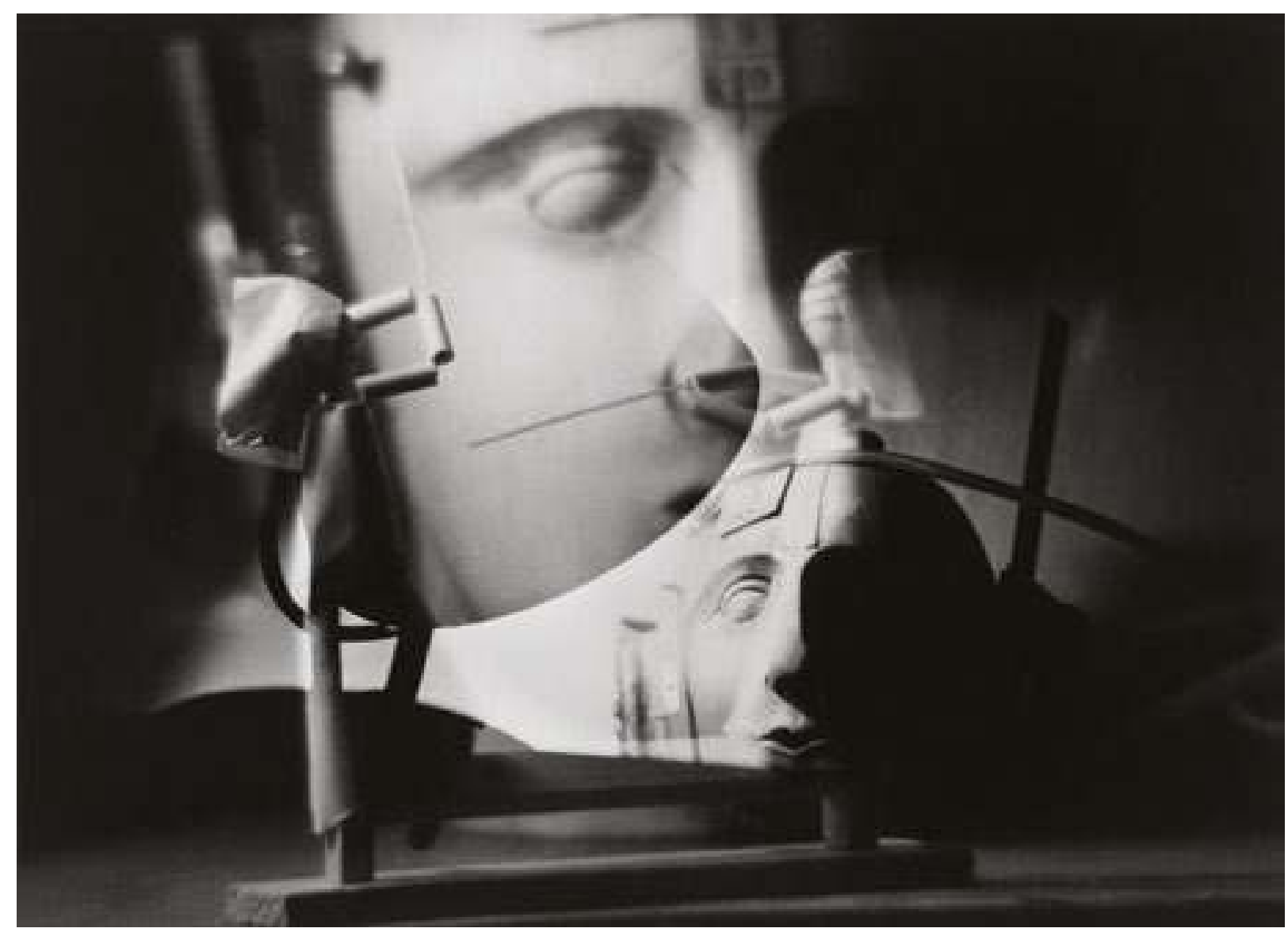

Fig. 3 Raoul Haussman, Jeux mécaniques, Limoges, 1957, surimpression épreuve gélatino-argentique $(21,4 \times 29,8 \mathrm{~cm}) \odot$ ADAGP, Paris $2011 \odot$ Collection Centre Pompidou, Dist. Rmn/Philippe Migeat.

«On peut élaborer des techniques pour s'adapter, voire apprendre la stratégie adverse, ce que j'ai exploré vers la fin. On essayait d'apprendre les stratégies adverses. Sinon, pour le contrôle de notre propre stratégie de jeu, on essayait de définir, avec cette vue globale, les rôles essentiels de chaque agent. S'il devait défendre, attaquer, faire une passe, et après dans la réalisation propre de chaque action pour chaque individu, on avait plutôt un calcul local et on retombait dans des modèles plus réactifs à base de champs, ou qui pouvaient être bio-inspirés. [...] On avait quand même déjà remarqué dans les premières compétitions que les équipes avaient des comportements efficaces, mais assez stéréotypés; du type : "on attaque, on frappe toujours la balle par les mêmes couloirs, les mêmes angles, les mêmes zones". On essayait d'apprendre les comportements les plus répétitifs au niveau de la position des robots. Typiquement là où ils frappent la balle pour frapper au but, les couloirs qu'ils utilisent pour faire ce mouvement-là, et d'arriver sur le temps d'une mi-temps, à dégager les lieux de l'espace où ils déclenchent leurs tirs, par exemple. Et l'idée, c'était qu'à la deuxième mitemps, on positionnait nos robots de défense exactement au milieu des zones qu'ils utilisent pour frapper, pour voir si ça les gênait beaucoup ou s'ils étaient capables de contourner et de s'adapter eux-mêmes. "

L'apprentissage constitue l'une des techniques parmi les plus employées dans le cadre d'une compétition de ce genre (Kitano 1997: 77-79). Il est, par ailleurs, tout à fait envisageable de construire un moteur stratégique qui fonde ses décisions à partir de l'utilisation d'un réseau de neurones artificiels (Laue 2008; Sukvichai 2009), ou encore de l'analyse de parties déjà jouées, placées dans la 


\section{PIÈGES À VOIR, PIÈGES À PENSER}

-

8. Rolf Pfeifer et Josh Bongard considèrent en effet que la RoboCup constitue l'un des meilleurs exemples de coopération entre agents. Ils considèrent, cependant, la coopération à partir d'une situation où le comportement des agents dépend de leur capacité, individuellement, à s'observer mutuellement et à déterminer leurs actes à partir de ces observations. II semble plutôt que ces matchs mettent en valeur la capacité d'un système à prendre des décisions en fonction (et pour contrer] des actions du système adverse. mémoire du système (Sukvichai 2009). D'autres outils, comme par exemple les champs conditionnels aléatoires (Zickler 2008), peuvent également entrer dans la conception d'un système capable d'encourager les robots à jouer ensemble, avec intelligence. C'est-à-dire d'une manière qui permette aux robots de se passer la balle, de se démarquer, de déterminer un angle de tir optimal et de marquer, ou d'empêcher la progression de l'adversaire, de bloquer les angles de tir, etc., tout cela en fonction des comportements des membres de l'équipe adverse, tels qu'ils sont analysés en temps réel par le système. C'est d'ailleurs là que semble résider toute la difficulté à penser ces systèmes robotiques en se référant au jeu ordinaire pratiqué par les humains.

Cette boucle systémique, aussi efficace qu'elle puisse être dans le cadre de la RoboCup, laisse en effet quelque peu douter du caractère réellement coopératif des robots, tel qu'il a notamment été mis en valeur par Pfeifer et Bongard ${ }^{8}$ (2007 : 226). Il est assez difficile d'imaginer que la coopération entre joueurs humains puisse se fonder sur une réactivité et une organisation tactique aussi centralisées. Dans le jeu de football ordinaire, le rôle de l'entraîneur ne consiste pas, en effet, à suivre la balle et à organiser le positionnement des joueurs en temps réel, de sorte qu'ils se placent dans les positions idéales de tir que l'entraîneur aura lui-même déduites d'un calcul de probabilités. Il consisterait plutôt en une forme de coopération et d'organisation collective, au sein de laquelle l'entraîneur et les membres de l'équipe entretiennent des relations où chacun dispose d'une réelle liberté d'initiative, et où les joueurs adaptent leur comportement en fonction $\mathrm{du}$ jeu des autres à partir du contrôle individuel des dispositions dans lesquelles chacun se trouve (Houseman 2003 : 299). Il est en fait assez délicat de parler, dans le cas de tels systèmes centralisés, d'une coopération fondée sur la base de comportements observés par chacun des robots en présence. Les boucles opérées par ces systèmes informatiques, à partir d'un modèle d'organisation stratégique, sont assez difficiles à saisir si on les considère seulement pour la continuité qu'elles visent à établir entre le jeu des humains et celui des machines. Il semble plus propice de les envisager à travers leurs limites. Comment cette intelligence présentée à travers le jeu serait-elle à la fois perçue et comprise par le public?

La focalisation des regards sur les actes des machines est généralement le support de réactions assez diverses parmi les spectateurs. Dans le cas de la Small Size League, ces réactions consistent le plus souvent à applaudir lorsqu'un but est marqué. L'intensité de ces applaudissements évolue en fonction de la qualité du système à tirer parti de situations de jeu délicates; si le système parvient par exemple à marquer en faisant circuler la balle au sein d'une défense compacte, ou encore si les deux équipes sont d'un niveau à peu près égal. Les spectateurs sont concentrés sur la manière dont le système fait coopérer les robots qui le composent. Les réactions de surprise ou les interjections qui sont parfois entendues autour du terrain, à la fois du côté des ingénieurs et de celui des spectateurs non spécialistes, interviennent dans les phases de jeu où les robots s'échangent la balle. Elles connotent une projection particulière de l'autonomie sur les machines là où, techniquement, celle-ci dépend exclusivement du système de commande. Toutefois, les actions de jeu ne semblent pas confondre les spectateurs sur la nature du jeu qu'ils observent, et donc créer une continuité par rapport au jeu que l'on cherche à représenter. Malgré la relative réussite d'un système à créer le sentiment de la coopération des actions de jeu qui sont pourtant le reflet de sa seule activité, l'action des machines elle-même reste difficilement comparable avec celle qu'elle tente de reproduire. Ainsi, les spectateurs les moins avisés 
conservent une certaine distance par rapport au spectacle qui leur est montré, observant l'action d'un air détaché, emprunt de curiosité, sans vraiment comprendre comment fonctionnent ces machines, mais en restant toutefois concentrés sur leur moindre déplacement. Certains d'entre eux esquissent parfois un sourire lorsqu'une machine adopte un comportement erratique, sort des limites du terrain, ou s'arrête sans raison apparente. Les plus prompts à réagir à l'action des robots sont les ingénieurs eux-mêmes. Ces derniers savent plus ou moins lire à travers l'activité des machines, et ils savent tirer de cette lecture les éléments d'une interprétation touchant à la qualité du travail accompli par chaque équipe. Mais dans les deux cas, du point de vue des spécialistes et de celui des nonspécialistes, l'action coordonnée des agents sur le terrain de jeu ne garantit pas la ressemblance des machines et des humains. Au mieux, permet-elle de renforcer le lien entre les ingénieurs et l'objet de leur travail. Dans les ligues humanoïdes, néanmoins, ce lien s'estompe jusqu'à confondre les roboticiens et les spectateurs à travers leur engagement dans le jeu qu'ils observent.

\section{Des comportements au modèle : une scénographie de l'effort}

Jusqu'à présent, nous avons évoqué des systèmes anthropomorphes placés au cœur d'affrontements qui s'offrent au public à travers l'action des machines. Nous avons noté que ces systèmes semblent d'abord échapper aux regards. Leur présence est liée aux logiciels utilisés depuis les marges du terrain, dans les ordinateurs des concurrents humains. Mais pour le public, ces systèmes se manifestent essentiellement à travers l'activité des agents robotiques. Le seul moyen, pour les spectateurs, d'évaluer la qualité du travail de chaque équipe humaine consiste à porter le regard sur l'action visible, et donc sur le jeu joué par les robots. Ces derniers relaient en quelque sorte l'activité invisible des concepteurs humains, à travers les décisions prises par le système de jeu. Cette forme de jeu pose ainsi ses limites. Une première concerne la qualité du modèle qui permet d'imiter le jeu réel. Une seconde touche à l'engagement des spectateurs dans la partie. Or si la Small Size League ne provoque pas de réaction très vive au sein du public, les deux grandes ligues humanoïdes ${ }^{9}$ forment un lieu où les spectateurs se manifestent avec plus d'enthousiasme. Évoquons deux souvenirs liés à un geste particulier.

Le premier de ces souvenirs concerne un match de Standard Platform League (fig. 4). Ce match opposa l'équipe qui, plus tard, remporta le titre, et une équipe au sein de laquelle on comprit rapidement que la seule performance que l'on pût espérer des robots fût qu'ils ne perdissent pas par un trop grand écart de points. À la fin de cette partie, l'un des ingénieurs de cette équipe leva les bras vers le ciel, comme si elle avait remporté le match, et s'écria : «Hourra! Nous n'avons perdu que sept-zéro! »Cette équipe n'avait pu lutter efficacement contre son adversaire, tant le système de jeu de ce dernier était performant.

$\mathrm{Au}$ sein des ligues humanoïdes, l'habitude veut que l'action des machines se fonde sur un traitement individuel des signaux. Contrairement à la Small Size League, où un seul modèle stratégique s'applique à tous les agents robotiques d'une équipe, les robots concourant dans les ligues humanoïdes fonctionnent sur le mode de la décentralisation, à partir de leur propre perception de l'environnement. Le jeu des robots articule ainsi une forme et des comportements. Chaque robot est conçu à partir de certaines fonctions visibles du corps humain. Ils sont bipèdes, possèdent des bras, et sont dotés de caméras. Toutefois, les modalités de

\footnotetext{
9. Ces deux ligues forment en fait trois groupes. Le premier, la Standard Platform League, fait concourir des robots Nao, conçus par la société française Aldebaran Robotics. Les deux autres groupes sont contenus dans la Humanoid League. Ici, chaque équipe doit concevoir ses robots. D'un côté, on trouve le groupe Kid Size, au sein duquel les machines ne doivent pas excéder soixante centimètres. De l'autre, on trouve le groupe Teen Size, où la taille des robots oscille entre un mètre et un mètre soixante.
} 
fonctionnement de ces éléments n'ont que peu à voir avec le fonctionnement des organes qu'ils imitent chez l'humain. Elles sont ainsi restreintes aux seules nécessités imposées par les limites techniques des machines et les règles de jeu. Le système de vision traite seulement certains éléments de l'environnement (comme la balle, les buts ou les lignes blanches du terrain), sans tenir compte ni de l'adversaire, ni du propre corps du robot. La bipédie ne permet pas aux machines de marcher à plus de quelques dizaines de centimètres par seconde. Et les bras sont principalement utilisés pour permettre à une machine de se relever après une chute. L'architecture interne des robots suit ces modalités de la perception, et oriente donc leur action en fonction de ce qui est perçu. Elle cherche à fonder non pas une intelligence collective, mais des capacités individuelles pour l'action. Cette condition de jeu donne des résultats assez décevants quant au type d'action offert aux spectateurs. Le plus souvent, lorsqu'ils ne passent pas leur temps à chercher la balle, les robots se dirigent tous vers elle, marchant péniblement, s'entrechoquant et finissant par tomber, déclenchant alors le rire des spectateurs.

Or, les vainqueurs de ce match étaient parvenus à une forme de coopération entre les machines, leur permettant de détecter les corps en présence, de communiquer et d'adapter leurs déplacements en fonction des éléments perçus dans le cours du match (Röfer 2009). L'équipe adverse, comme les autres équipes de cette ligue, n'avait pas prévu d'avoir à lutter contre une telle organisation stratégique. Cependant, elle avait conçu un geste particulier pour son gardien de but, qui l'aida, non pas à remporter la victoire, mais à s'attirer la sympathie d'une grande partie du public et des ingénieurs eux-mêmes. À un moment de cette partie perdue d'avance, un robot adverse s'avança lentement vers la balle pour la frapper. Tous semblaient prêts à assister à un nouveau but. Mais cette fois, le gardien se trouvait dans une position qui lui permit de détecter la balle. Au moment où le robot déclencha son tir, le gardien se propulsa sur ses pieds et, tendant le bras au-dessus de sa tête, tomba lourdement sur le sol. Se plaçant ainsi sur la trajectoire de la balle, il en dévia la course et sauva ainsi son camp, le temps d'une action. Pendant que le robot se redressait, l'ensemble de l'équipe poussa un cri de joie, partagé par le public, enthousiasmé par une telle action de jeu. Un homme âgé, assis à mes côtés, frappait des mains et il cria plus fort encore que le reste de la foule amassée devant ce spectacle. «Voilà, s'exclama-t-il, ça c'est du football! » Que penser alors d'une telle forme de focalisation sur l'action des machines? Du point de vue humain, on sait qu'un geste peut consister en une unité minimale de communication (Kendon 2004), qu'il peut porter le contenu d'un message (Ekman et Friesen 1969 : 55-56). Mais qu'en serait-il d'un geste technique qui, effectué par un objet, n'aurait d'autre fin que le but qu'il vise? Les actes des robots humanoïdes vaudraient-ils, dans l'œil d'un spectateur, pour les indices de l'intelligence qui les pousse à agir?

"Parce que l'objet automatisé "marche tout seul", écrit Jean Baudrillard, il impose une ressemblance avec l'individu humain autonome, et cette fascination l'emporte. " (1968: 157) Le geste d'un gardien de but robotisé semble bien imposer une certaine continuité avec le geste d'un gardien de but humain. Il en possède l'aspect, et cette forme, insérée dans l'environnement particulier d'un terrain de football, est aisément reconnaissable par les spectateurs. Ce geste est, par ailleurs, effectué dans le cadre d'une action de jeu, et consiste en une réaction automatique du système de commande interne du robot. En réalité, l'intelligence d'un tel geste est d'abord à mettre sur le compte de celle qui l'a rendu possible. Question d'échelle, elle est certes le résultat de ce qui se joue au sein même de la machine, 


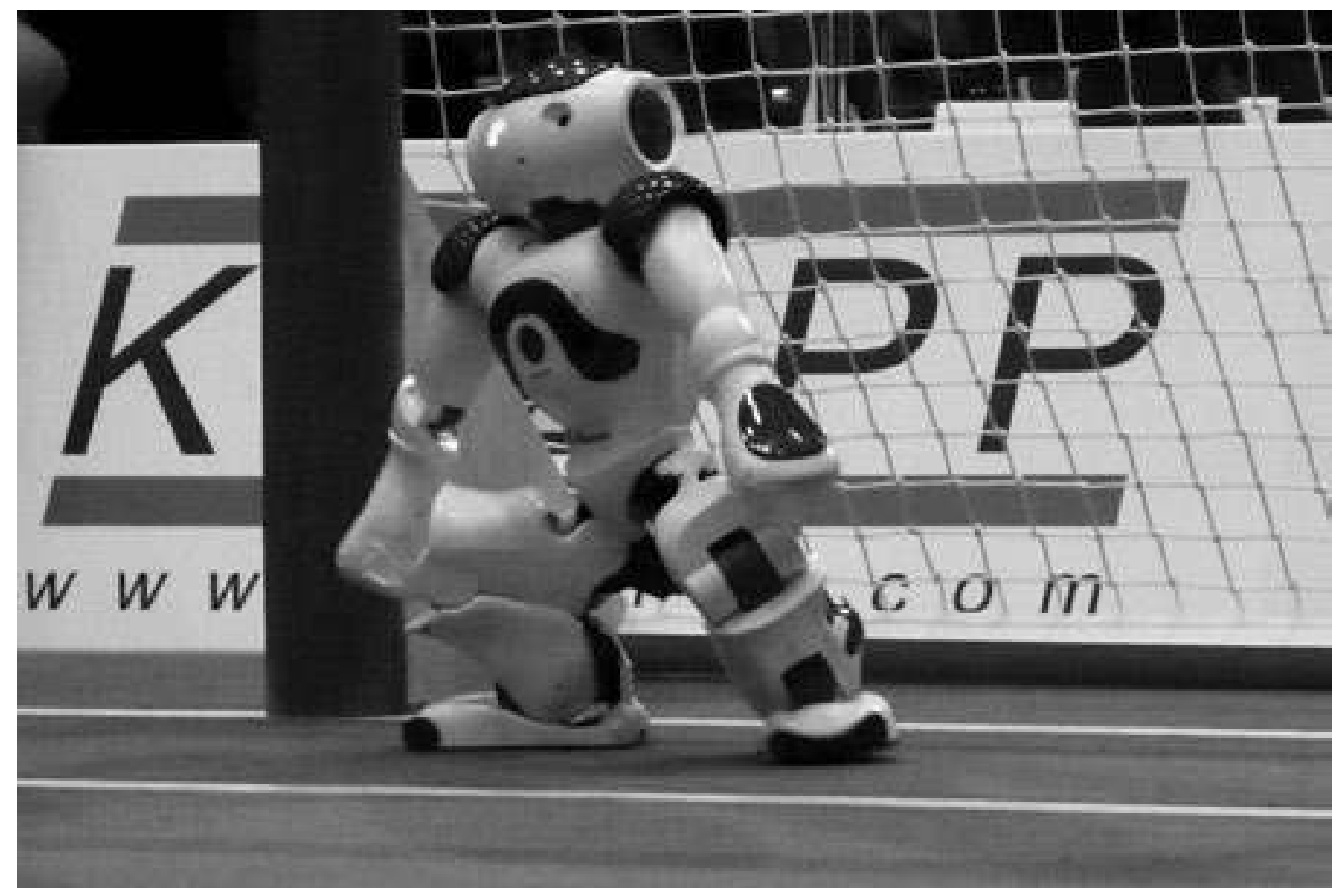

Fig. 4 Un robot Nao occupe le rôle de gardien de but lors d'un match de Standard Platform League, RoboCup 2009, Graz. Photo Joffrey Becker.

et consiste ainsi en une boucle entre perception et action, mais elle résulte surtout de l'activité de l'humain qui lui a donné forme. À ce niveau de l'observation, le travail de l'ingénieur consiste en une activité d'automatisation de l'animation même de la machine par la constitution d'une intériorité spécifique. Celle-ci, insérée dans le cadre particulier de la partie, produit un autre niveau d'observation où la machine, seule, rend compte des capacités de l'ingénieur à analyser et construire une stratégie performante. L'efficacité de cette stratégie demeure cependant liée ici à l'efficacité du geste perçu. Or, écrit Edwin Carels, l'animation découle toujours d'une interaction entre une machine et la perception humaine (2010 : 63). L'animation, poursuit-il, est aussi un format à part entière qui active et fonctionne à travers une esthétique combinatoire, reliant les cadres individuels afin d'encourager l'expérience de mouvements n'existant que dans l'esprit du spectateur (Carels 2010 : 67). Les liaisons entre le corps humain et les machines prendraient ici un aspect particulier, touchant aux principes de codification et de déchiffrement de l'image tels qu'ils se construisent à travers la mimesis (Gombrich 1972; 2002), en d'autres termes, à travers un processus de construction collective de l'image.

Dans ce cas, la continuité s'instaure à plusieurs niveaux. Au niveau de l'ingénieur, elle permet de penser la méthode avec laquelle celui-ci construit les capacités d'action des machines par imitation d'une activité humaine, et adaptation du produit de cette imitation à la morphologie particulière de l'objet ou du système d'objets. Au niveau des machines, elle fonde une relation directe, icono- 
- 1

10. C'était du moins le cas lors de la RoboCup 2009. Les règles ont été modifiées pour l'édition 2010. graphique et ordinaire (Grimaud 2008:41), entre les éléments que l'ingénieur a gardé du réel et ce que la machine peut accomplir. Au niveau des spectateurs, enfin, elle permet une inférence particulière où le geste accompli vaut pour le geste imité. Les machines se constituent ainsi en des formes d'illustrations de l'activité des humains à partir du redoublement de quelques-uns de leurs comportements ordinaires de jeu. Elles offriraient ainsi de renforcer la mécanicité du corps même de l'humain dont elles empruntent les façons d'agir, par un jeu de projections croisées sur l'objet à travers ses actions. Mais il y a plus, lorsque l'on songe à ces entrelacements des aspects formels des images du corps et des machines. Dans l'activité sportive humaine, souligne Georges Vigarello, la technicisation du corps de l'athlète, qui participe des fondations du rapport moderne du corps et de la machine, induit une relation inédite avec la motricité, et une tension paradoxale entre la prévision et la surprise: "Soumission aux règles maximales d'efficacité biomécanique d'abord, selon un calcul sophistiqué de vecteurs, de forces, de durées, mais aussi attention toujours plus avivée aux ratages et aux imprévus ensuite, ceux que la pratique ludique ne peut manquer de comporter. » (Vigarello $2006: 171$ )

Ces imprévus fondent, dans le cas des jeux de machines, une certaine ambiguïté qu'un second exemple nous permettra d'envisager. Déplaçons-nous vers un autre match. Celui-ci a lieu dans le cadre de la Humanoid League, et plus précisément dans le groupe Teen Size. Ce groupe oppose des prototypes de robots humanoïdes bipèdes et prend principalement la forme de duels ${ }^{10}$. Ces duels opposent un robot situe au centre du terrain et un autre robot situé dans les buts. L'arbitre dépose le ballon derrière le robot du centre et donne un coup de sifflet. On déclenche alors le jeu. Le robot doit, dans un temps limité, localiser la balle, se diriger vers elle et tirer vers le but. Le robot adverse, lui, doit se positionner sur sa ligne de but pour intercepter le ballon. Le plus souvent, la machine cherche à se positionner sur la trajectoire de la balle en effectuant de petits pas de côté. Mais ce n'est pas la stratégie conçue pour le robot qui retiendra notre attention. Celuici a été fabriqué de telle sorte à plonger et intercepter la balle. Son armature a été renforcée avec de la mousse et il peut donc se jeter au sol sans trop risquer de se briser. Ce geste, que nous avons déjà évoqué plus haut comme étant le principe d'une continuité avec son modèle, prend ici un aspect inattendu. Nous sommes au début d'un match opposant deux équipes qui s'affronteront à nouveau en finale. Le robot retenant notre attention se trouve dans ses cages lorsque l'arbitre dépose la balle derrière son adversaire. Il vient de se lever après que l'un de ses
Fig. 5 Replacement défensif des robots de l'équipe B-Smart concouran. RoboCup 2009, Graz. Photo Joffrey Becker.

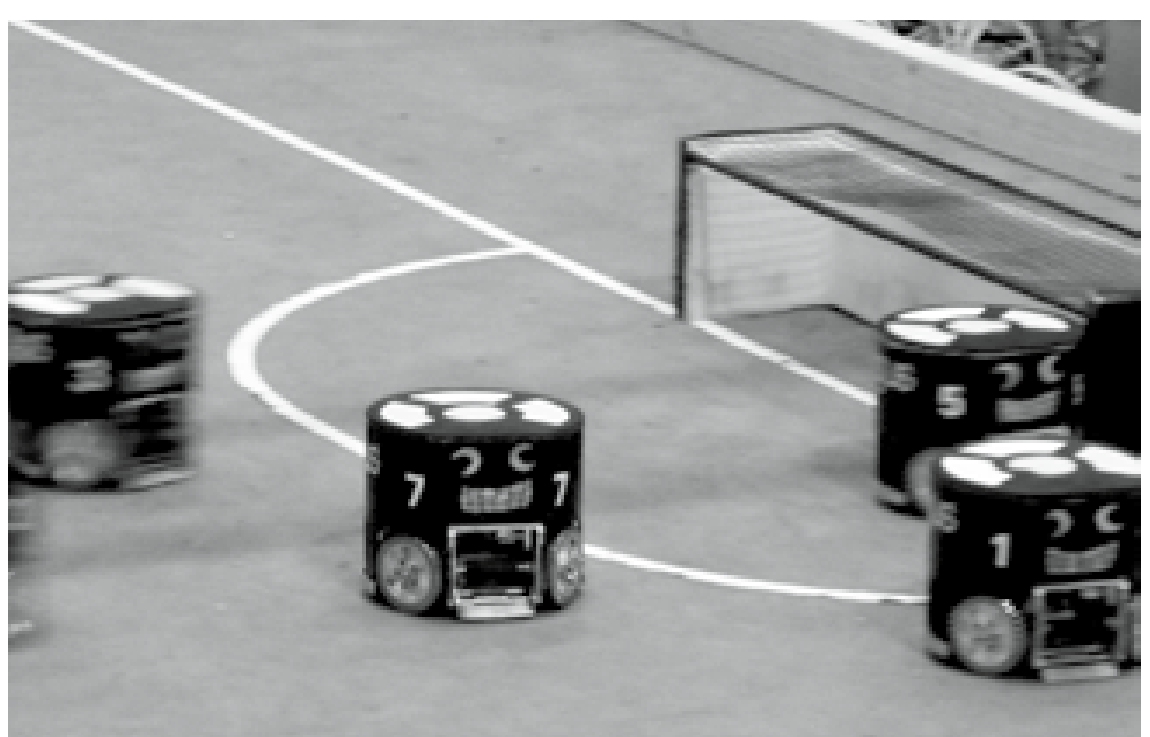


concepteurs l'a rapidement fait passer du mode attaquant au mode gardien. Son adversaire, immobile, se tient également prêt. Les ingénieurs quittent alors le terrain tandis que quelques personnes s'arrêtent, voyant qu'un match est sur le point de commencer. L'arbitre lance la partie. Le coup de sifflet retentit. Alors que son adversaire commence seulement à chercher à déterminer la position de la balle d'un mouvement de tête, subitement, le robot se jette au sol, les deux bras levés, sans raison apparente. Les quelques spectateurs présents autour du terrain éclatent alors de rire. Les créateurs de ce robot, eux, dissimulent mal leur gêne. Responsables de cette erreur, ils ne pouvaient plus intervenir sur cette machine gisant bras tendus, immobile sur le sol, tandis que son adversaire tentait péniblement de trouver la balle. Ce dernier finit par se placer dans une position de tir, et frappa la balle à la limite du temps réglementaire. Malheureusement pour ses concepteurs, il tira sur le corps inerte de son adversaire.

Cette scène reflète assez bien les situations de jeu plus habituelles, traversées par l'erreur ou l'approximation; ces mêmes situations qui déclenchent, ailleurs, l'hilarité des spectateurs. Le rire consiste, dans ces compétitions, en une réaction aussi ordinaire que peuvent l'être les applaudissements. On pense alors à ce qu'en a dit Bergson (2007); car au fond, ce qui amuse en pareil cas, c'est d'abord l'apparent manque d'adresse de la machine, ce qui, précisément, la rendrait mécanique. Une telle maladresse ne semble en effet pouvoir trouver d'équivalence dans un comportement de jeu humain. Nul gardien de but humain ne pourrait commettre une telle faute. Ce genre d'erreur, qui s'illustre à travers l'action des machines, consiste bien en un comportement spécifique de ces dernières, qui révèle d'une manière inattendue le lien très étroit liant les opérateurs à leurs objets (l'erreur de l'objet révélant celle de l'opérateur), et joue sur l'ambiguïté de la distance entre l'objet et son modèle (le rire du modèle révélant cette ambiguïté). Laissé là par le rire, dans cet état d'objet inerte, le robot semble néanmoins doté d'une autonomie nouvelle, qui va au-delà de celle-là même dont l'ont doté ses concepteurs, et paradoxalement lui confère un aspect proprement humain. Cet écart participerait en effet d'un redoublement de la tension, à l'œuvre dans le jeu, entre précision et approximation. Or, cette tension semble trouver un certain écho parmi les spectateurs, qui peuvent observer dans les actes des machines des éléments de comportements ordinaires. Ceux-ci, soumis à cet impératif du jeu, peuvent être traversés autant par la technicité du geste que par ses approximations et ses erreurs. Cette tension entre l'implicite et l'explicite du jeu permet en fait de mettre en valeur des relations particulières entre les humains à travers l'action des robots.

Lorsque l'imitation d'une forme humaine comprend une modalité particulière de la perception, une certaine continuité avec l'humain semble pouvoir se former. Mais c'est aussi lorsqu'un robot existe indépendamment de la volonté de son concepteur, à travers l'ambiguïté qui caractérise ses actes, qu'il peut être considéré comme autonome, du point de vue des spectateurs non spécialistes comme des ingénieurs. À travers un jeu dont la profondeur se forme ainsi sur l'alternance entre leur adresse et leur maladresse, les robots touchent à la remémoration d'une image du corps que nul ne semble pourtant être en mesure de reconstituer complètement. Les actions de l'objet, telles qu'elles s'agencent automatiquement en séquences de jeu, ne constituent pas pour autant un principe suffisant pour valider le modèle, mais plutôt un espace d'incertitudes au sein duquel le caractère de l'objet dépend précisément d'un jeu de projections parcellaires et contradictoires. 


\section{Récursions chimériques}

Dans un tel contexte, ces machines peuvent être envisagées comme des objetschimères (Severi 2007). Car, en cristallisant des éléments formels et comportementaux, renvoyant à des régimes d'existence divers, et dont l'image générale ne peut se résumer à l'addition de celles qu'elle agrège, les robots anthropomorphes mobilisent la mémoire des spectateurs à travers un exercice perceptif et interprétatif touchant à ce que l'image contient d'implicite. Ces machines peuvent alors pénétrer l'espace chimérique grâce à l'autonomie particulière que leurs actes permettent d'imputer. En effet, l'autonomie ne comprend pas qu'une question de programmation. Elle émergerait d'abord de l'écart, matériel et formel, qui caractérise toute tentative de figuration d'un modèle naturel (Marin 1994), mais également les formes de lectures que ces tentatives permettent (Gombrich 2002). D'un côté, les ingénieurs déduisent les actions des robots à partir de l'ensemble des problèmes qu'ils se doivent de résoudre et des règles qu'ils ont à suivre. De l'autre, les spectateurs induisent sur ce qu'ils perçoivent de l'environnement, de la forme des objets qui y évoluent et des actions de ces derniers, les éléments d'une interprétation. Les propos de Hans Moravec, que nous citions en introduction, soulignent que la conséquence de l'utilisation conjointe de la déduction et de l'induction favorise l'apparition d'une nouvelle catégorie d'existant (1988: 50), prennent un sens nouveau lorsqu'on se place au niveau des relations, ici complémentaires, permettant de construire l'image des robots. C'est là un second lieu de l'autonomie, qui se situe au niveau de la focalisation sur des éléments clés des systèmes, et dont l'action est située dans un environnement et un temps particuliers. Les mouvements des robots offrent, en effet, aux spectateurs de concentrer leur attention - et donc de fonder leur interprétation - sur les éléments mobiles du dispositif; c'est-à-dire de leur permettre de projeter du sens sur ces éléments à travers quelques points de référence (Wagner 1986). Ce type de jeu consiste pourtant en un redoublement de l'activité même de jeu; où le jeu lui-même vaut pour le jeu dont il assure la représentation. L'autonomie émergerait alors de la mise en abîme des caractères de continuité du jeu lorsqu'il se donne à voir. Ces caractéristiques ont pour principal effet de placer les joueurs humains dans une position symétrique par rapport à celle des spectateurs, les transformant en supporters de l'intelligence dont ils sont à l'origine. Dans ce cadre, cependant, on peut penser que le robot vaille autant pour un double fictionnel permettant à l'humain de se penser en tant que tel (Moisseeff 2008 : 155), que pour le reflet des idées de ses concepteurs, et du regard particulier qu'ils posent sur le corps de l'humain qui joue, et dont ils tentent de redoubler l'image. Mais tous les spectateurs ne disposent pas de l'ensemble des éléments qui fondent cette étroite relation.

On peut effectivement penser que cette autonomie nouvelle des objets, qu'elle se forme de manière complémentaire entre la déduction et l'induction, ou de manière symétrique lorsque les machines se donnent en spectacle, mobilise des croyances très diverses, où la récursivité liée aux fonctions naturelles de l'action de jeu interviendrait comme une façon de gérer l'écart entre le modèle et son image. Car au fond, si les spectateurs ont à juger du degré de continuité avec l'objet qui, d'une certaine manière, les représente, ils le font à partir de ses capacités d'action, telles qu'elles sont pensées en termes de fonction. S'ils rendent compte de la forme du vivant par leurs mouvements, comme les automates des premières heures de la modernité (Bredekamp 1995 : 46-51), les systèmes et leurs robots procèdent d'une illustration d'après nature, où la vie peut se résumer à certaines 


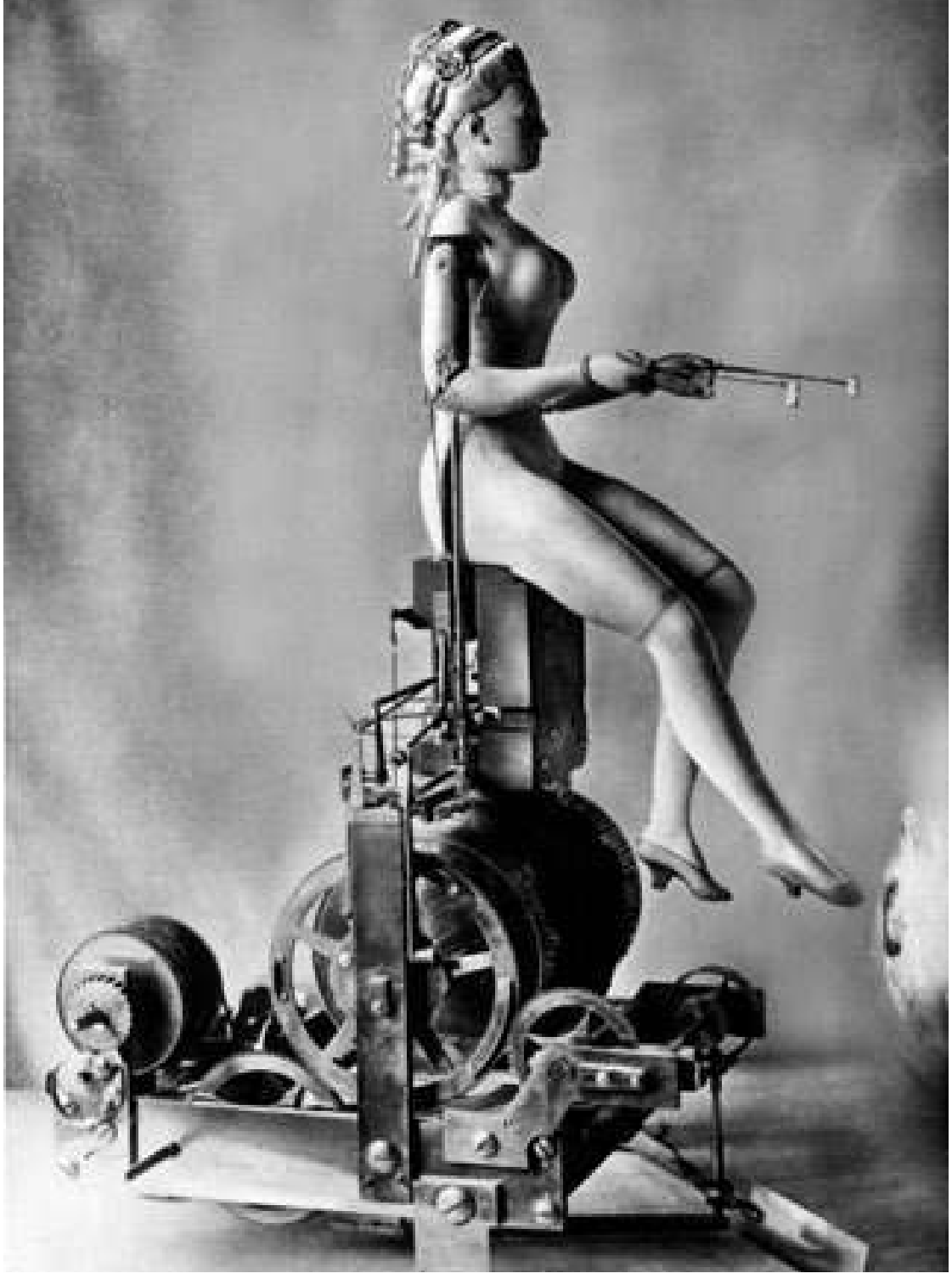

Fig. 6 Joueuse de clavecin. Automate appartenant à Marie-Antoinette, poupée mécanique. vers 1930 ๑ BPK, Berlin, Dist. Rmn/Image BPK.

fonctions élémentaires qui témoigneraient, mieux que tout discours, de la mécanicité du vivant. Cette prééminence de la notion de mécanique dans l'indistincte fonctionnalité du vivant, son extraction sous la forme de modèles, son implantation dans l'inertie de la matière, puis l'effet de vie que cette dernière semble, en retour, faire naître au sein du public, par le biais du mouvement, mais également des tentatives maladroites et des erreurs, participent ainsi d'une relation cyclique qui trouve d'étonnantes résonances avec l'activité symbolique (Sperber 2008; Vidal 2007). Ces relations métamorphosent la fonction en substrat à partir duquel peut se fonder l'image d'un corps-machine, obligé de passer sans cesse à l'état d'objet pour mieux être envisagé en tant qu'humain, et à l'inverse, de revenir vers la vie pour mieux être envisagé en tant que machine. Tel serait finalement le jeu contenu dans cette imitation ludique d'un jeu. À condition, toutefois, que ce jeu-ci en soit encore un.

EHESS, Laboratoire d'anthropologie sociale joffrey.becker@ehess.fr

mots clés / keywords: chimères // chimeras - robotique // robotics • anthropomorphisme // anthropomorphism • performance // performance. 


\section{Bibliographie}

\section{Bateson, Gregory}

1977 [1972] «Une théorie du jeu et du fantasme », Vers une écologie de l'esprit. T. I. Paris, Seuil : 247-264.

\section{BAUDRILLARD, Jean}

1968 «Le système méta- et dysfonctionnel : gadgets et robots », Le Système des objets. Paris, Gallimard : 151-188.

\section{Bergson, Henri}

$200 ?$ [1900] Le Rire. Essai sur la signification du comique. Paris, Presses universitaires de France.

\section{BraitenBERG, Valentino}

1991 Véhicules. Expériences en psychologie synthétique. Lausanne, Presses polytechniques et universitaires romandes.

\section{BrEAzEAL, Cynthia}

2003 « Toward sociable robots », Robotics and autonomous systems 42(3-4) : 167-175.

\section{BREDEKAMP, Horst}

1995, The Lure of Antiquity and the Cult of the Machine. Princeton, Markus Wiener Publishers.

\section{BRooks, Rodney}

2002 Flesh and Machines. How Robots Will Change Us. New York, Vintage Books.

\section{CARELs, Edwin}

2010 « Biometry and antibodies, Modernizing animation / Animating modernity », in Anselm Franke (éd.), Animism. Vol. I. Berlin-New York, Sternberg Press : 57-74.

\section{Crevier, Daniel}

1997 [1993] « Jeux : échec et mat pour les machines ? », À la recherche de l'intelligence artificielle. Paris, Flammarion : 257-279.

EkMan, Paul et Friesen, Wallace V. 1969 « The repertoire of nonverbal behavior: Categories, Origins, Usage, and Coding », Semiotica 1(1) : 49-98.

\section{GeerTz, Clifford}

2005 « Deep play: notes on the Balinese cockfight », Daedalus 134(4) : 56-86.

\section{Gelin, Rodolphe}

2006 Le Robot, ami ou ennemi ? Paris, Le Pommier.

\section{GeLl, Alfred}

1992 « The technology of enchantement and the enchantement of technology », in Jeremy Coote et Anthony Shelton (éd.), Anthropology, Art and Aesthetics. Oxford, Clarendon Press : 40-63.

\section{GOMBRICH, Ernst H.}

1972 «Action and expression in western art », in Robert A. Hinde [éd.], Non-Verbal Communication. Cambridge, Cambridge University Press : 373-395.

2002 [1960] L'Art et l'lllusion. Psychologie de la représentation picturale. Paris, Phaidon.

\section{GRIMAUd, Emmanuel}

2008 «L'hypnose mécanique ou le bougé comme unité du vivant », Dieux et robots. Le théâtre des automates divins de Bombay. Paris, L'Archange Minotaure : 36-47.

\section{Guillot, Agnès et Meyer, Jean- Arcady} 2008 La Bionique. Quand la science imite la nature. Paris, Dunod.

\section{Houseman, Michael}

2003 « Vers un modèle anthropologique de la pratique thérapeutique », Thérapie familiale 24[3] : 289-312.

\section{Kendon, Adam}

2004 « Gesture units, gesture phrases and speech », Gesture. Visible Action as Utterance. Cambridge, Cambridge University Press : 108-126.

KitAno, Hiroaki, et al.

1997 « RoboCup: A challenge problem for Al », Al Magazine 18[1] : 73-95.

Laue, Tim, et al. 2008 B-Smart (Bremen Small Multi-Agent Robot Team], Team description for RoboCup 2008. Bremen, Universität Bremen.

\section{MARIN, Louis}

1994 «Mimésis et description 》, De la représentation. Paris, Gallimard-Seuil : 252-266.

\section{Moisseeff, Marika}

2008 « Nous n'avons jamais été humains. Le néotène, les chimères et les robots », in Serge Gruzinski (éd.), Planète métisse. Paris, Actes Sud-musée du quai Branly : 152-164.

\section{Moravec, Hans}

1988 Mind children. The future of robot and human intelligence. Cambridge, Harvard University Press.

\section{Mori, Masahiro}

1970 «The uncanny valley », Energy ? (4) : 33-35.

\section{Pfeifer, Rolf et Bongard, Josh}

2007 How the Body Shapes the Way We Think.

A new view on intelligence. Cambridge, MIT Press.

\section{Riskin, Jessica}

2003 « The defecating duck, or the ambiguous origins of artificial life », Critical inquiry 29(4) : 599-633.

\section{RÖFER, Thomas, et al.}

2009 B-Human: Team report and code release 2009. Brème, Universität Bremen.

\section{SeVeri, Carlo}

2007 Le Principe de la chimère. Une anthropologie de la mémoire. Paris, Éditions Rue d'Ulm-musée du quai Branly [《AEsthetica 》].

2009 «La parole prêtée. Comment parlent les images », in Carlo Severi et Julien Bonhomme (dir.), Paroles en actes. Paris, L'Herne

[«Cahiers d'anthropologie sociale 5 »] : 11-41.

\section{Sperber, Dan}

2008 [1974] Le Symbolisme en général. Paris, Hermann.

Sukvichai, Kanjanapan, et al. 2009 Skuba 2009 Team Description. Bangkok, Kasetsart University.

\section{VIDAL, Denis}

2007 « Anthropomorphism or SubAnthropomorphism? An anthropological Approach to Gods and Robots 》, Journal of the Royal Anthropological Institute 13 : 917-933.

\section{Vigarelio, Georges}

2006 «S'entraîner », in Jean-Jacques Courtine [éd.], Histoire du corps. Les mutations du regard, le xxe siècle. Vol. III. Paris, Seuil : 163-197.

WAGNER, Roy

1986 Symbols that Stand for Themselves. Chicago, University of Chicago Press.

Zickler, Stefan, et al. 2008 CMDragons 2008 Team Description. Pittsburgh, Carnegie Mellon University. 


\section{Résumé / Abstract}

Joffrey Becker, Récursions chimériques. Del'anthropomorphisme des robots autonomes à l'ambiguïté de l'image du corps humain - L'une des caractéristiques des créatures artificielles se fonde sur un rapport de continuité et d'indépendance vis-à-vis des modèles dont elles empruntent l'aspect. La robotique autonome ne semble pouvoir échapper à cette relation entre imitation et invention. En cherchant ainsi à préciser sa conception mécaniste du corps par la production d'objets spécifiques, cette forme récente de robotique induit une transformation qui confond, dans les objets qu'elle crée, des régimes symboliques d'existence relevant pourtant d'ontologies bien distinctes. Nous explorerons d'abord quelques aspects formels du transfert de ces éléments d'ontologie aux objets. Nous tenterons alors de comprendre comment, à travers leurs actes, les robots permettent de figurer une image du corps humain. Nous nous appuierons sur une série d'analogies construites par les roboticiens dans le cadre d'une compétition de machines autonomes.
Joffrey Becker, Chimeric Recursion. From the anthropomorphism of autonomous robots to the ambiguity of images of the human body-One of the characteristics of artificial entities is the way in which they embody relationships of both continuity and independence vis-a-vis the models whose likeness they assume. Indeed, autonomous robotics seems unable to escape this tension between imitation and invention. Its efforts to translate a mechanistic conception of the body into specific objects have led to a situation where these objects hypostasize different symbolic regimes of existence (associated with different ontologies). This article begins by exploring several formal aspects of this process by which ontological elements are transferred to objects. It then seeks to understand how robotic actions give body to an image of the human form. It makes use of a series of analogies employed by roboticians during a competition between autonomous robots. 